\title{
Anthropometric evaluation of pre-school and school age children from Azores archipelago, Portugal
}

\author{
Avaliação antropométrica de crianças com idade pré-escolar e escolar do arquipélago dos \\ Açores, Portugal
}

\author{
Cíntia Ferreira-Pêgo ${ }^{1}$, Ana Carrapa ${ }^{2}$, Sara Gaipo ${ }^{3}$, Tânia Parece ${ }^{3}$, Raquel Marinho ${ }^{3}$, Tiago Dias ${ }^{3}$, Fred- \\ erico Viveiros ${ }^{4}$, Rita Carvalho ${ }^{5}$, Rui César ${ }^{5}$, Nelson Tavares ${ }^{1}$ \\ ${ }^{1}$ CBIOS Lusófona's Research Center for Biosciences and Health Technologies, Av. Campo Grande 376, 1749-024 \\ Lisbon, Portugal. \\ ${ }^{2}$ Unidade de Saúde da Ilha Terceira, Angra do Heroísmo, Açores \\ ${ }^{3}$ Unidade de Saúde da Ilha de S. Miguel, S. Miguel, Açores \\ ${ }^{4}$ Unidade de Saúde da Ilha do Faial, Açores \\ ${ }^{5}$ Serviço de Endocrinologia e Nutrição, Hospital do Divino Espirito Santo, S. Miguel, Açores \\ Email: nelson.tavares@ulusofona.pt
}

\begin{abstract}
Childhood obesity is associated with immediate adverse consequences, such as lower self-esteem and lower educational attainment, and a higher risk for many harmful comorbidities later in life, such as type 2 diabetes mellitus, dyslipidemia, non-alcoholic fatty liver disease, hypertension, and coronary heart disease.

A cross-sectional analysis to assess the prevalence of normal weight, overweight, and obesity in pre-school and school children from Azores archipelago was designed. The final sample size included 1069 participants (546 boys and 523 girls) aged between 3.00 and 9.99 years old.

Significant differences were found when the analysis was performed considerating overweight and obese as the same category, demonstrating that girls were heavier than boys. Also, girls presented higher BMI than boys specifically at age 6,7 , and 9 years old. The odds ratio of presenting overweight or obese were significantly higher for girls and participants with 4, 6, and 9 years old. Finally, children from the island of Faial demonstrated a lower prevalence of overweight and/or obesity when considering the island of São Miguel as a reference. Our results reveal a high prevalence of obesity mainly among female participants, heightening the need for interventions at early ages, mainly in this population, to effectively control childhood obesity in Portugal.
\end{abstract}

Keywords: obesity, overweight, children, Azores, Portugal

\begin{abstract}
Resumo
A obesidade infantil está associada a consequências adversas imediatas, como menor autoestima e menor nível educacional, assim como maior risco de comorbidades na idade adulta.

Foi levado a cabo uma análise transversal para avaliar a prevalência de peso normal, excesso de peso e obesidade em crianças em idade pré-escolar e escolar do arquipélago dos Açores. A amostra incluiu 1069 participantes (546 rapazes e 523 raparigas) com idades entre 3,00 e 9,99 anos.

Quando a análise foi realizada considerando o excesso de peso e obesidade na mesma categoria, foram encontradas diferenças significativas, demonstrando que as raparigas eram mais pesadas que os rapazes. Além disso, as meninas apresentaram maior IMC do que os meninos aos 6,7 e 9 anos. O Odds Ratio de apresentar excesso de peso ou obesidade foi significativamente maior para raparigas e participantes com 4, 6 e 9 anos. Por fim, crianças da ilha do Faial apresentaram uma menor prevalência de excesso de peso e/ou obesidade, tendo em consideração a ilha de São Miguel como referência. Os nossos resultados revelam uma alta prevalência de excesso de peso e/ou obesidade principalmente entre participantes do sexo feminino, aumentando a necessidade de intervenções em idades precoces, para controlar efetivamente a obesidade infantil em Portugal.
\end{abstract}

Palavras-chave: obesidade, excesso de peso, crianças, Açores, Portugal 


\section{Introduction}

Obese children are more likely to become obese adults and to develop significant health consequences at younger ages $(1,2)$. Childhood obesity is associated with immediate adverse consequences, such as psychological problems and lower educational attainment, and a higher risk for many harmful comorbidities later in life, such as type 2 diabetes mellitus, dyslipidemia, non-alcoholic fatty liver disease, hypertension, and coronary heart disease (3-7).

Adipose tissue is considered a complex and highly active metabolic endocrine organ (8), producings various cytokines (9) involved in the regulation of energy metabolism, inflammation, diabetes and atherosclerosis (10-12). Increased levels of adipokines and proinflammatory cytokines have prominent roles in the pathogenesis of the metabolic syndrome (9).

For all these reasons, obesity is a growing health concern that has become an epidemic in modern-day society (13). In particular, childhood obesity has increased dramatically in the past decades $(14,15)$, and if this trend is not reversed, the number of overweight or obese infants and young children worldwide is predicted to double in less than a decade (16).

Prior research suggests that the Portuguese trend of childhood obesity prevalence is plateauing, following the overall pattern for developed countries (17-19). Nevertheless, a recent article from 2018 shows rising trends in overweight (including obesity) in children from 4 through 10 years of age, contrary to these others reports (20). It is important to note that Portugal remains among the top six European countries with the highest prevalence of childhood obesity, with $31.6 \%$ overweight (including obese) children aged 7-8 years (17). Additionally, the archipelago of the Azores is the area of Portugal with the highest levels of obesity showing 35.9\% (30) which could bring innumerous health consequences to several generations.

Considering all of these reasons, the main objective of the present analyses was to evaluate the anthropometric data from children aged between 3 and 9 years old from Azorean archipelago and the prevalence of normal weight, overweight, and obese- of the same sample.

\section{Introdução}

Crianças obesas têm maior probabilidade de se tornarem adultos obesos, assim como de desenvolver consequências significativas para a saúde em idades mais jovens $(1,2)$. A obesidade infantil está associada a efeitos adversos imediatos, como problemas psicológicos e menor nível educacional, além de um risco maior de muitas comorbidades nocivas durante a idade adulta, como Diabetes Mellitus Tipo 2, dislipidemia, doença hepática não alcoólica, hipertensão e doenças coronárias (3-7).

O tecido adiposo é uma fonte bem conhecida de inflamação e considerado um órgão endócrino metabólico complexo e altamente ativo (8). Produz várias citoquinas (9) que estão envolvidas na regulação do metabolismo energético, inflamação, diabetes e aterosclerose (10-12). Os níveis aumentados de citoquinas pró-inflamatórias têm um papel proeminente na patogénese da síndrome metabólica (9).

Por todas essas razões, a obesidade é uma preocupação crescente de saúde e que se tornou uma epidemia na sociedade moderna (13). Em particular, a obesidade infantil aumentou drasticamente nas últimas décadas $(14,15)$ e, se essa tendência não for revertida, prevê-se que o número mundial de bebés e crianças com excesso de peso ou obesidade duplique em menos duma década (16).

Pesquisas anteriores sugeriram que a tendência portuguesa da prevalência da obesidade infantil estabilizou, seguindo o padrão geral para os países desenvolvidos (17-19). No entanto, um artigo recentemente publicado em 2018, mostra tendências crescentes de excesso de peso (incluindo obesidade) em crianças dos 4 aos 10 anos de idade, contrariando esses anteriores relatórios (20). É importante ressalvar que Portugal permanece entre os seis principais países europeus com maior prevalência de obesidade infantil, com $31,6 \%$ das crianças com 7 a 8 anos de idade apresentando excesso de peso (incluindo obesidade) (17). É importante também ressalvar que o arquipélago dos Açores é a área de Portugal com os níveis de obesidade mais altos (21), (30) um número chocante que poderá trazer inúmeras consequências para a saúde de várias gerações.

Tendo em consideração todos os motivos descrito anteriormente, o principal objetivo da presente análise foi avaliar os dados antropométricos de crianças entre os 3 e os 9 anos de idade do arquipélago dos Açores e posteriormente avaliar a prevalência de peso normal, excesso de peso e obesidade da mesma amostra. 


\section{Material and Methods}

\section{Design and study population}

The present study is a cross-sectional analysis designed to assess the prevalence of normal weight, overweight, and obese in children aged between 3.00 and 9.99 years old. The participants were recruited from public schools from São Miguel, Faial and Terceira Islands, all of them included in the Azores archipelago. The children's inclusion criteria involved parental consent (following the Declaration of Helsinki) (29), having no apparent illness, and to be present on the scheduled visit day. The evaluations took place during the 2018/2019 school year. The effective sample size of the present work was 1069 participants.

\section{Anthropometric parameters assessment}

Trained personnel measured weight using an electronic scale, recording the value to the nearest decigram (0.1 $\mathrm{kg}$ ), and height using a calibrated stadiometer. Height was assessed with the children standing upright, with their backs to the stadiometer and with their feet and knees together, their head positioned so that the gaze remained horizontal, according to the Frankfurt plane. The value was recorded to the nearest millimeter $(0.1$ $\mathrm{cm})$. All the participants wore light clothing and no shoes during the measurements. Body Mass Index (BMI) was calculated from the measurements obtained in each child using the normalized formula [Weight (in $\mathrm{kg}$ )/ Height ${ }^{2}$ (in m)] (19). Normal weight, overweight and obese were classified using the international age and gender cut-off points according to those proposed by the International Obesity Task Force (IOTF) (22).

\section{Statistical analysis}

Data are presented either as means and standard deviation (SD) for continuous variables or numbers and percentages for dichotomous variables. We compared the distribution of the selected characteristics between groups using Pearson $\chi^{2}$ tests for categorical variables or Student's t-tests or analysis of variance (ANOVA), as appropriate, for continuous variables. Logistical regression models were fitted to assess the associations between presenting BMI corresponding to overweight and/or obesity cutoff (dependent variable) and gender (two categories), age (years), age categories (seven categories) or island (three categories) as exposure. The models were adjusted for gender, age and island, ex-

\section{Material e Métodos}

\section{Desenho e estudo da população}

O presente estudo é uma análise transversal desenvolvido para avaliar a prevalência de peso normal, excesso de peso e obesidade em crianças com idade entre os 3,00 e os 9,99 anos de idade. Os participantes foram recrutados aleatoriamente em escolas públicas das ilhas de São Miguel, Faial e Terceira, todas incluídas no arquipélago dos Açores. Os critérios de inclusão das crianças envolveram o consentimento por escrito dos encarregados de educação (de acordo com a Declaração de Helsínquia) (29), não apresentar doença aparente e estar presente no dia agendado para a visita. As avaliações ocorreram durante o ano letivo de 2018/2019. O tamanho efetivo da amostra do presente trabalho foi de 1069 participantes.

\section{Avaliação dos dados antropométricos}

O peso foi medido por profissionais treinados utilizando uma balança eletrónica, registando o valor no decigrama mais próximo $(0,1 \mathrm{~kg})$ e a altura usando um estadiómetro calibrado. A estatura foi avaliada com as crianças em pé, de costas para o estadiómetro e com os pés e joelhos juntos, a cabeça posicionada para que o olhar permanecesse horizontal, de acordo com o plano de Frankfurt. $\mathrm{O}$ valor foi registrado no milímetro mais próximo $(0,1 \mathrm{~cm})$. Todos os participantes usaram roupas leves e não usaram sapatos durante todas as medições. O Índice de Massa Corporal (IMC) foi posteriormente calculado a partir das medidas obtidas em cada criança, utilizando a fórmula normalizada [Peso $(\mathrm{em} \mathrm{kg}) /$ Altura $^{2}$ (em m)] (19). Peso normal, excesso de peso e obesidade foram classificados usando os pontos de corte internacionais de idade e sexo, de acordo com os propostos pelo International Obesity Task Force (IOTF) (22).

\section{Análise estatística}

Os dados são apresentados como médias e desvio padrão (DP) para variáveis contínuas ou números (n) e percentagens (\%) para variáveis dicotómicas. Comparámos a distribuição das características selecionadas entre os grupos usando testes de Pearson $\chi^{2}$ para variáveis categóricas ou testes $t$ de Student ou análise de variância (ANOVA), conforme apropriado, para variáveis contínuas. Os modelos de regressão logística foram ajustados para avaliar as associações entre apresentar excesso de peso e/ou obesidade (variável dependente) e sexo (duas categorias), idade (anos), categorias de idade (sete categorias) ou ilha (três categorias) como exposição. Os modelos foram ajustados para sexo, idade 
cept when any of these variables were the independent variable. All statistical tests were two-tailed and the significance level was set at $p<0.05$. All analyses were performed using the SPSS software version 22.0 (SPSS Inc, Chicago, IL).

\section{Results}

A total of 546 boys and 523 girls (1069 participants) from Azores archipelago with a mean age of 5.62 years (SD:1.87) took part in the present analysis. Children from S. Miguel Island are 803, from Terceira Island 184 and 82 from Faial Island. The general characteristics of the studied population according to gender are summarized in Table 1. No statistical differences were found regarding age, height, weight, and BMI in the studied population.

Table 2 shows the anthropometric measurements for each age categories assessed, separated by gender. Boys had a significantly higher average height than the girls at ages 4 and 5 . No other differences were observed in additional parameters, i.e., weight, BMI, or different age categories.

Median BMI for each age category is shown in Figure e ilha, exceto quando qualquer dessas variáveis fosse a variável independente. Todos os testes estatísticos foram bicaudais e o nível de significância foi estabelecido em $\mathrm{P}<0,05$. Todas as análises foram realizadas utilizando o software SPSS versão 22.0 (SPSS Inc, Chicago, IL).

\section{Resultados}

Participaram na presente análise 546 rapazes e 523 raparigas (1069 participantes) do arquipélago dos Açores, com uma idade média de 5,62 anos (DP: 1,87). As características gerais da amostra de acordo com o sexo estão resumidas na Tabela 1. Não foram encontradas diferenças estatísticas na população estudada em relação à idade, altura, peso e IMC.

A tabela 2 mostra as medidas antropométricas para cada faixa etária, separadas por sexo. Os rapazes apresentaram uma estatura média significativamente mais elevada que as raparigas aos 4 e 5 anos de idade. Não foram observadas mais diferenças em relação a outros parâmetros adicionais, como peso ou IMC, nem noutras categorias de idade.

A média de IMC para cada categoria de idade pode ser

Table 1/ Tabela 1 - General characteristics of the study population categorized by gender./ Características gerais da população estudada categorizada por sexo.

\begin{tabular}{|c|c|c|c|c|}
\hline & $\begin{array}{l}\text { All population/ } \\
\text { População total } \\
(\mathrm{n}=1069)\end{array}$ & $\begin{array}{c}\text { Boys/ } \\
\text { Rapazes } \\
(n=546)\end{array}$ & $\begin{array}{c}\text { Girls/ } \\
\text { Raparigas } \\
(\mathrm{n}=523)\end{array}$ & $\begin{array}{c}\text { p- } \\
\text { value/ } \\
\text { valor } \\
\text { es-p }^{\mathbf{a}}\end{array}$ \\
\hline Age/ Idade, years/ anos & $5.62(1.87)$ & $5.53(1.85)$ & $5.71(1.89)$ & 0.122 \\
\hline Height/ Altura, m & $1.13(0.13)$ & $1.13(0.13)$ & $1.13(0.13)$ & 0.984 \\
\hline Weight/ Peso, kg & $22.75(8.16)$ & $22.66(8.04)$ & $22.84(8.28)$ & 0.711 \\
\hline $\mathrm{BMI} / \mathrm{IMC}, \mathrm{kg} / \mathrm{m}^{2}$ & $17.22(3.06)$ & $17.18(3.08)$ & $17.28(3.05)$ & 0.611 \\
\hline \multicolumn{5}{|c|}{$\begin{array}{l}\text { Age categories/ Categorias de } \\
\text { idade, } \%(n)\end{array}$} \\
\hline 3 years/ anos & $13.10(140)$ & $14.30(78)$ & $11.90(62)$ & \\
\hline 4 years/ anos & $20.90(223)$ & $20.70(113)$ & $21.00(110)$ & \\
\hline 5 years/ anos & $20.60(220)$ & $21.20(116)$ & $19.90(104)$ & \\
\hline 6 years/ anos & $10.80(115)$ & $11.50(63)$ & $9.90(52)$ & 0.641 \\
\hline 7 years/ anos & $15.10(161)$ & $13.90(76)$ & $16.30(85)$ & \\
\hline 8 years/ anos & $10.30(110)$ & $9.90(54)$ & $10.70(56)$ & \\
\hline 9 years/ anos & $9.40(100)$ & $8.40(46)$ & $10.30(54)$ & \\
\hline
\end{tabular}

Data expressed as means (SD) or percentages (n), as appropriate. Abbreviations: BMI, body mass index. a p-values for comparisons between groups were tested by Student's t-test or Pearson $\chi^{2}$ as appropriate. / Dados expressos em média (DP) ou percentagens (n). Abreviaturas: IMC, Índice de Massa Corporal. a valores-p para comparações entre grupos foram testados mediante teste $t$ de Student ou Pearson $\chi 2$ como apropriado. 
Table 2/ Tabela 2 - Anthropometric measurements according to gender and age categories./ Tabela 2. Dados antropométricos de acordo com o sexo e categorias de idade.

\begin{tabular}{|c|c|c|c|c|}
\hline & $\begin{array}{l}\text { All population/ } \\
\text { População total }\end{array}$ & $\begin{array}{l}\text { Boys/ } \\
\text { Rapazes }\end{array}$ & $\begin{array}{c}\text { Girls/ } \\
\text { Raparigas }\end{array}$ & $\begin{array}{c}\mathrm{p} \text {-value/ } \\
\text { valores-p }^{\mathrm{a}}\end{array}$ \\
\hline 3 years/ anos & $(\mathrm{n}=140)$ & $(\mathrm{n}=78)$ & $(n=62)$ & \\
\hline Height/ Altura & $0.95(0.05)$ & $0.95(0.05)$ & $0.95(0.04)$ & 0.716 \\
\hline Weight/ Peso & $15.30(2.87)$ & $15.41(3.00)$ & $15.16(2.71)$ & 0.618 \\
\hline $\mathrm{BMI} / \mathrm{IMC}$ & $16.92(2.43)$ & $17.01(2.62)$ & $16.81(2.17)$ & 0.642 \\
\hline 4 years/ anos & $(\mathrm{n}=223)$ & $(n=113)$ & $(n=110)$ & \\
\hline Height/ Altura & $1.03(0.05)$ & $1.04(0.53)$ & $1.02(0.05)$ & 0.005 \\
\hline Weight/ Peso & $18.09(3.46)$ & $18.44(3.58)$ & $17.73(3.31)$ & 0.124 \\
\hline $\mathrm{BMI} / \mathrm{IMC}$ & $16.94(2.37)$ & $16.94(2.27)$ & $16.93(2.48)$ & 0.976 \\
\hline 5 years/ anos & $(n=220)$ & $(n=116)$ & $(n=104)$ & \\
\hline Height/ Altura & $1.10(0.06)$ & $1.12(0.05)$ & $1.09(0.06)$ & 0.003 \\
\hline Weight/ Peso & $20.25(4.07)$ & $20.71(4.44)$ & $19.74(3.57)$ & 0.074 \\
\hline $\mathrm{BMI} / \mathrm{IMC}$ & $16.52(2.51)$ & $16.58(2.78)$ & $16.45(2.18)$ & 0.717 \\
\hline 6 years/ anos & $(\mathrm{n}=115)$ & $(n=63)$ & $(n=52)$ & \\
\hline Height/ Altura & $1.17(0.06)$ & $1.18(0.07)$ & $1.16(0.05)$ & 0.169 \\
\hline Weight/ Peso & $23.69(5.66)$ & $23.92(5.74)$ & $23.42(5.60)$ & 0.637 \\
\hline $\mathrm{BMI} / \mathrm{IMC}$ & $17.21(2.99)$ & $17.14(2.81)$ & $17.30(3.23)$ & 0.773 \\
\hline 7 years/ anos & $(n=161)$ & $(n=76)$ & $(\mathrm{n}=85)$ & \\
\hline Height/ Altura & $1.23(0.06)$ & $1.23(0.07)$ & $1.23(0.06)$ & 0.857 \\
\hline Weight/ Peso & $25.99(6.23)$ & $25.87(6.26)$ & $26.09(6.23)$ & 0.830 \\
\hline $\mathrm{BMI} / \mathrm{IMC}$ & $17.08(2.97)$ & $16.98(2.88)$ & $17.17(3.06)$ & 0.680 \\
\hline 8 years/ anos & $(n=110)$ & $(n=54)$ & $(n=56)$ & \\
\hline Height/ Altura & $1.28(0.06)$ & $1.28(0.06)$ & $1.28(0.06)$ & 0.589 \\
\hline Weight/ Peso & $28.93(7.00)$ & $29.24(7.67)$ & $28.62(6.35)$ & 0.643 \\
\hline $\mathrm{BMI} / \mathrm{IMC}$ & $17.50(3.32)$ & $17.56(3.49)$ & $17.45(3.17)$ & 0.863 \\
\hline 9 years/ anos & $(n=100)$ & $(n=46)$ & $(n=54)$ & \\
\hline Height/ Altura & $1.34(0.07)$ & $1.34(0.07)$ & $1.34(0.07)$ & 0.965 \\
\hline Weight/ Peso & $35.94(10.55)$ & $35.41(11.06)$ & $36.40(10.18)$ & 0.644 \\
\hline $\mathrm{BMI} / \mathrm{IMC}$ & $19.80(4.64)$ & $19.52(4.97)$ & $20.04(4.37)$ & 0.577 \\
\hline
\end{tabular}

Data expressed as means (SD). Height expressed in meters (m), weight in kilograms $(\mathrm{kg})$ and BMI in $\mathrm{kg} / \mathrm{m}^{2}$. Abbreviations: BMI, body mass index. a $\mathrm{p}$-values for comparisons between groups were tested by Student's t-test./ Dados expressos como media (DP). Altura expressa em metros $(\mathrm{m})$, peso em quilogramas $(\mathrm{kg})$ e IMC em kg/m². Abreviaturas: IMC, Indice de Massa Corporal. a valores-p para comparações entre grupos foram testados mediante teste $t$ de Student. 
1. Girls presented higher BMI than boys at ages 6, 7 and 9 years old. At 4 and 8 years old, both girls and boys presented BMI values very similar to each other.

The prevalence of (being) overweight and obese in female children was higher in all age categories (non-significant) (except for ages 7 and 8) than male children (Table 3$)$. Statistically significant differences $(\mathrm{p}=0.034)$ were found in the total population (not split by age categories) considering overweight and obese as the same category (overweight and/or obese vs. normal weight individuals), girls $35.00 \%$ and boys $28.90 \%$ (data not shown).

The odds ratios of presenting BMI higher than IOTF overweight cutoff for children (Table 4) were significantly higher for girls and participants with 4, 6 and 9 years old in the fully-adjusted model. When the comparison was performed by gender, it was found that boys aged 6 and boys and girls aged 9 years presented a higher risk of being overweight and/or obese. In the same line of research, the odds ratio analyses by island was also performed. Children from the island of Faial presented a lower prevalence for overweight and/or obese, considering São Miguel Island as a reference. No additional significant results were observed for the other island or when the analyses were performed split by gender. encontrada na Figura 1. As raparigas apresentaram IMC superior aos rapazes aos 6, 7 e 9 anos de idade. Tanto as raparigas como os rapazes com 4 e 8 anos de idade, apresentaram valores de IMC muito semelhantes entre si. As crianças do sexo feminino apresentaram (diferenças não significativas), em todas as categorias de idade, maior prevalência de excesso de peso e obesidade (exceto para os 7 e 8 anos) que as crianças do sexo masculino (Tabela 3). Foram encontradas diferenças significativas $(\mathrm{p}=0,034)$ em relação à população total (não subdividida por categorias de idade) quando a análise foi realizada considerando o excesso de peso e a obesidade na mesma categoria (indivíduos com sobrepeso e/ou obesidade vs. peso normal), apresentando as raparigas $35,00 \%$ e os rapazes $28,90 \%$ (dados não mostrados).

A Odds Ratio de apresentar IMC superior ao ponto de corte recomendado pela IOTF para excesso de peso (Tabela 4) foi significativamente maior para as raparigas e participantes com 4, 6 e 9 anos, no modelo mais ajustado. Quando a comparação foi realizada por sexo, verificou-se que os rapazes de 6 anos, assim como os meninos e meninas de 9 apresentaram maior risco de estar acima do peso recomendado. Na mesma linha de pesquisa, também foram realizadas as análises de Odds Ratio por ilha. As crianças da ilha do Faial apresentaram menor prevalência de excesso de peso e/ou obesidade, tendo em consideração a ilha de São Miguel como referência. Não foram observados outros resultados significativos adicionais.

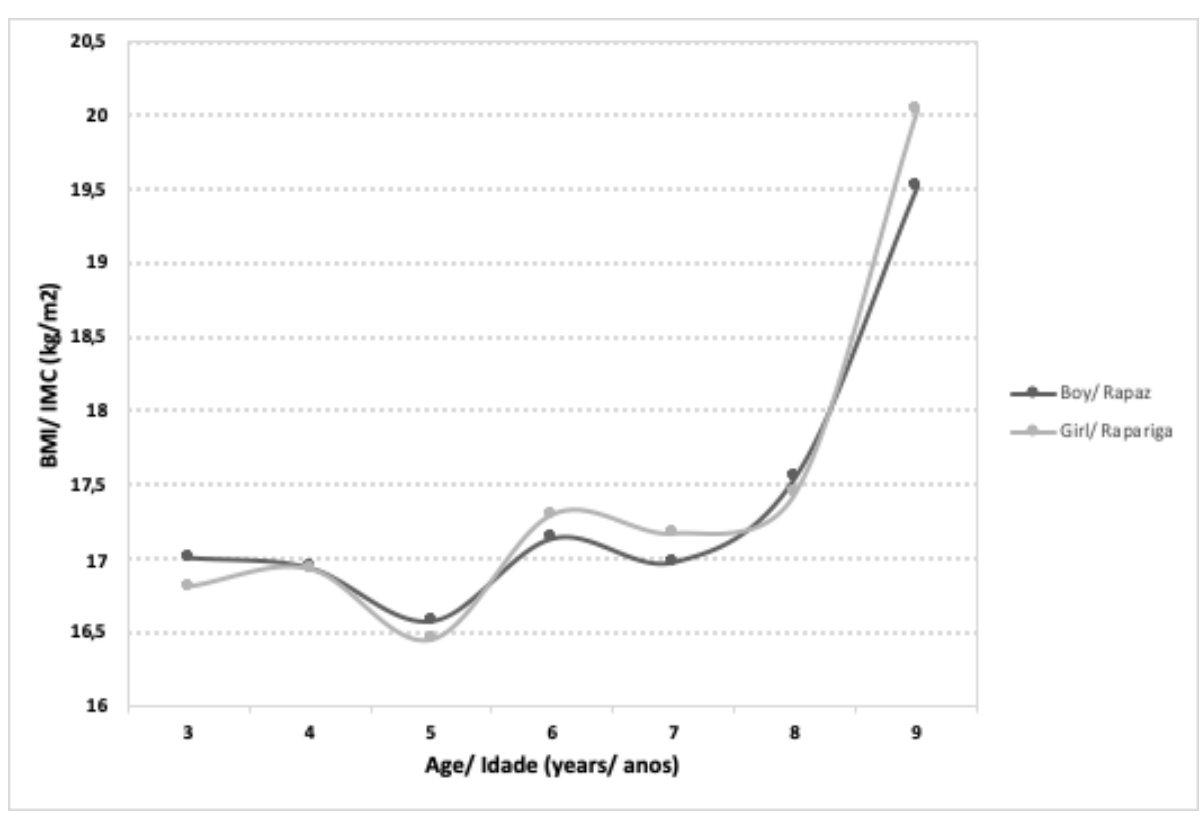

Figure 1/ Figura 1 - Median BMI values for each age categories by gender./ Valores médios de IMC para cada categoria de idade por sexo. 
Table 3/ Tabela 3 - Prevalence of normal weight, overweight and obesity according to gender and age categories./ Prevalência de peso normal, excesso de peso e obesidade de acordo com o sexo e categorias de idade.

\begin{tabular}{|c|c|c|c|c|}
\hline & $\begin{array}{l}\text { All population/ } \\
\text { População total }\end{array}$ & $\begin{array}{c}\text { Boys/ } \\
\text { Rapazes }\end{array}$ & $\begin{array}{c}\text { Girls/ } \\
\text { Raparigas }\end{array}$ & $\begin{array}{c}\mathrm{p} \text {-value/ } \\
\text { valores-p }\end{array}$ \\
\hline \multicolumn{5}{|l|}{3 years/ anos } \\
\hline Normal Weight/ Peso normal & $76.40(107)$ & $79.50(62)$ & $72.60(45)$ & \multirow{3}{*}{0.618} \\
\hline Overweight/ Excesso de peso & $14.30(20)$ & $12.80(10)$ & $16.10(10)$ & \\
\hline Obesity/ Obesidade & $9.30(13)$ & $7.70(6)$ & $11.30(7)$ & \\
\hline \multicolumn{5}{|l|}{4 years/ anos } \\
\hline Normal Weight/ Peso normal & $66.80(149)$ & $67.30(76)$ & $66.40(73)$ & \multirow{3}{*}{0.990} \\
\hline Overweight/ Excesso de peso & $18.80(42)$ & $18.60(21)$ & $19.10(21)$ & \\
\hline Obesity/ Obesidade & $14.30(32)$ & $14.20(16)$ & $14.50(16)$ & \\
\hline \multicolumn{5}{|l|}{5 years/ anos } \\
\hline Normal Weight/ Peso normal & $73.60(162)$ & $77.60(90)$ & $69.20(72)$ & \multirow{3}{*}{0.279} \\
\hline Overweight/ Excesso de peso & $13.60(30)$ & $10.30(12)$ & $17.30(18)$ & \\
\hline Obesity/ Obesidade & $12.70(28)$ & $12.10(14)$ & $13.50(14)$ & \\
\hline \multicolumn{5}{|l|}{6 years/ anos } \\
\hline Normal Weight/ Peso normal & $60.00(69)$ & $63.50(40)$ & $55.80(29)$ & \multirow{3}{*}{0.589} \\
\hline Overweight/ Excesso de peso & $19.10(22)$ & $15.90(10)$ & $23.10(12)$ & \\
\hline Obesity/ Obesidade & $10.90(24)$ & $20.60(13)$ & $21.20(11)$ & \\
\hline \multicolumn{5}{|l|}{7 years/ anos } \\
\hline Normal Weight/ Peso normal & $70.80(114)$ & $73.70(56)$ & $68.20(58)$ & \multirow{3}{*}{0.195} \\
\hline Overweight/ Excesso de peso & $19.90(32)$ & $14.50(11)$ & $24.70(21)$ & \\
\hline Obesity/ Obesidade & $9.30(15)$ & $11.80(9)$ & $7.10(6)$ & \\
\hline \multicolumn{5}{|l|}{8 years/ anos } \\
\hline Normal Weight/ Peso normal & $67.30(74)$ & $68.50(37)$ & $66.10(37)$ & \multirow{3}{*}{0.911} \\
\hline Overweight/ Excesso de peso & $16.40(18)$ & $14.80(8)$ & $17.90(10)$ & \\
\hline Obesity/ Obesidade & $16.40(18)$ & $16.70(9)$ & $16.10(9)$ & \\
\hline \multicolumn{5}{|l|}{9 years/ anos } \\
\hline Normal Weight/ Peso normal & $53.00(53)$ & $58.70(27)$ & $48.10(26)$ & \multirow{3}{*}{0.465} \\
\hline Overweight/ Excesso de peso & $22.00(22)$ & $21.70(10)$ & $22.20(12)$ & \\
\hline Obesity/ Obesidade & $25.00(25)$ & $19.60(9)$ & $29.60(16)$ & \\
\hline \multicolumn{5}{|l|}{ TOTAL } \\
\hline Normal Weight/ Peso normal & $68.10(728)$ & $71.10(388)$ & $65.00(340)$ & \multirow{3}{*}{0.070} \\
\hline Overweight/ Excesso de peso & $17.40(186)$ & $15.00(82)$ & $19.90(104)$ & \\
\hline Obesity/ Obesidade & $14.50(155)$ & $13.90(76)$ & $15.10(79)$ & \\
\hline
\end{tabular}

Data expressed as percentages (n). ${ }^{\text {a }} \mathrm{p}$-values for comparisons between groups were tested by Pearson $\chi 2$. Normal weight, overweight or obesity were categorized according to IOTF criteria (22). / Dados expressos como percentagem (n). ${ }^{a}$ valores-p para comparações entre grupos foram testados mediante Pearson $\chi 2$. Peso normal, excesso de peso e obesidade foram categorizados de acordo com os critérios da IOTF (22). 
Table 4/ Tabela 4 - Association (odds ratio) between presenting overweight and/or obesity (BMI) $>25 \mathrm{~kg} / \mathrm{m}^{2}$ and gender or age categories./

Associação (odds ratio) entre apres entar excesso de peso e/ou obesidade $\mathrm{IMC}>25 \mathrm{~kg} / \mathrm{m}^{2}$ e sexo ou categorias de idade.

\begin{tabular}{|c|c|c|c|}
\hline Variables/ Variáveis & $\begin{array}{l}\text { All population/ } \\
\text { População total } \\
(n=1069)\end{array}$ & $\begin{array}{l}\text { Boys/ } \\
\text { Rapazes } \\
(n=546)\end{array}$ & $\begin{array}{l}\text { Girls/ Raparigas } \\
\quad(\mathrm{n}=523)\end{array}$ \\
\hline Gender/Sexo ${ }^{a}$ & $1.32(1.01-1.72)$ & - & - \\
\hline \multicolumn{4}{|c|}{$\begin{array}{l}\text { Age categories/ Categorias } \\
\text { de idade }\end{array}$} \\
\hline 3 years/ anos & 1.00 (ref.) & 1.00 (ref.) & 1.00 (ref.) \\
\hline 4 years/ anos & $1.66(1.01-2.73)$ & $1.95(0.97-3.91)$ & $1.41(0.69-2.90)$ \\
\hline 5 years/ anos & $1.16(0.71-1.91)$ & $1.13(0.56-2.28)$ & $1.19(0.58-2.44)$ \\
\hline 6 years/ anos & $2.25(1.30-3.88)$ & $2.31(1.08-4.94)$ & $2.18(0.99-4.81)$ \\
\hline 7 years/ anos & $1.37(0.81-2.31)$ & $1.50(0.70-3.21)$ & $1.25(0.60-2.58)$ \\
\hline 8 years/ anos & $1.60(0.91-2.81)$ & $1.86(0.84-4.16)$ & $1.38(0.63-3.05)$ \\
\hline 9 years/ anos & $2.90(1.66-5.08)$ & $2.90(1.29-6.55)$ & $2.86(1.31-6.23)$ \\
\hline \multicolumn{4}{|l|}{ Island/ Ilha } \\
\hline São Miguel & 1.00 (ref.) & 1.00 (ref.) & 1.00 (ref.) \\
\hline Faial & $0.50(0.29-0.88)$ & $0.49(0.22-1.09)$ & $0.52(0.24-1.11)$ \\
\hline Terceira & $0.89(0.59-1.33)$ & $0.93(0.51-1.71)$ & $0.85(0.49-1.48)$ \\
\hline
\end{tabular}

Data expressed as ORs (95\% Confidence Interval). a Gender was coded as 0 for male and 1 for female. Logistic regression model adjusted for gender, age and island (except when any of these variables were the independent variable)./ Dados expressos em OR (95\% intervalo de confiança): ${ }^{a}$ Sexo foi codificado como 0 para masculino e 1 para feminino. Modelo de regressão logística ajustada por sexo, idade e ilha (exceto quando algumas dessas variáveis foram analisadas como variáveis independentes).

\section{Discussion}

The results of the present study indicate that $31.90 \%$ of the total population presented overweight, $14.50 \%$ of these being obese. The WHO European COSI reports suggested that childhood obesity in Portugal is slightly decreasing, with a drop from $15.30 \%$ (2008) to $13.90 \%$ (2013), without major sex differences $(17,18)$. In our sample, the estimated prevalence of obesity was higher when compared to the last results of the WHO European COSI surveys (14.50\% vs. $13.90 \%$ ), suggesting that Portuguese children remain among the most overweight and obese across Europe. Specifically, some of the highest levels of obesity in the world are found in island populations (23-27), as the case of Azores. These results can be explained by a range of factors, including low levels of physical activity and a decrease in the consumption of the traditional foods of the islands, such as fresh fish, meat, and local fruits and vegetables, which have been replaced with a high-energy-dense

\section{Discussão}

Os resultados do presente estudo indicam que 31,90\% da população total apresentou excesso de peso, sendo $14,50 \%$ obesos. Os relatórios europeus do Child Obesity Surveillance Initiative (COSI)/Organização Mundial da Saúde (OMS) Europa sugeriram que a obesidade infantil em Portugal está a diminuir levemente, com uma queda de 15,30\% (em 2008) para 13,90\% (em 2013), sem grandes diferenças em relação a sexo $(17,18)$. Na nossa amostra, a prevalência estimada de obesidade foi maior quando comparada com os últimos resultados das pesquisas europeias COSI/OMS (14,50\% vs. 13,90\%), sugerindo que as crianças portuguesas permanecem entre as que apresentam maiores níveis de excesso de peso e obesidade de toda a Europa. Especificamente, alguns dos níveis mais elevados de obesidade no mundo são encontrados em populações insulares (23-27), como é o caso dos Açores. Esses resultados podem ser explicados por uma série de fatores, incluindo baixos 
diet (27). Data from 6- to 10-year-old children from the nine islands of the archipelago of Azores (28) showed very similar results to those found in our study, as the prevalence of overweight in girls was $36.00 \%$ and in boys was $29.90 \%$, which was associated with low levels of physical activity, particularly in girls.

Girls were also significantly more overweight than boys (without age distinction) and presented a higher risk of having higher than recommended BMI levels according to IOTF criteria. The results observed in the present analyses were in line with other previous reports $(15,18,19,28)$, where the authors described that girls showed a higher prevalence of overweight (including obesity) in early/middle childhood.

Regarding the lower prevalence of excess weight and/ or obesity found in individuals from Faial Island compared to those from São Miguel, to the best of our knowledge, the present publication is the first one to describe this finding. Further studies are needed to better understand the sociodemographic variables that could explain it.

In Table 4 it was verified that the 4, 6, and 9 year-old individuals were those who presented higher prevalences related to being overweight according IOTF criteria than the children of the remaining age levels. These differences do not follow any chronological order and for this reason, are difficult to explain. However, it was also previously reported in Portugal that at age 4 and 7 girls presented statistically higher BMI values than boys, and at age 10 boys were heavier than girls (21). These results suggest that it is possible that some relation exists between being overweight and age, mainly at 4, 6-7 and 9-10 years old.

Furthermore, we observed that children were more prone to increase their BMI at age 4, 6 and 9 years, indicating that this is the best period to strengthen obesity prevention strategies. It is imperative to highlight the importance of prevention and reinforce the need for effective early age population-based strategies to prevent children from initially becoming overweight.

\section{Conclusion}

Girls presented higher BMI values than boys at age 6, 7 and 9 years. Girls also presented higher levels of overweight and obesity than boys, without age differ- níveis de atividade física e uma diminuição no consumo de alimentos tradicionais, frescos e locais das ilhas, como peixe, carne e frutas e legumes, que foram substituídos por uma dieta com uma alta densidade energética (27). Dados de crianças de 6 a 10 anos das nove ilhas do arquipélago dos Açores (28) mostraram resultados muito semelhantes aos encontrados por nós, uma vez que a prevalência de excesso de peso nas meninas foi de $36,00 \%$ e nos meninos foi de $29,90 \%$, os quais foram associados a baixos níveis de atividade física, principalmente nas raparigas.

Na presente analise, as raparigas também apresentaram um excesso de peso significativamente maior do que os rapazes (sem distinção de idade) e apresentaram maior risco de apresentar níveis de IMC acima do recomendado. Estes resultados observados foram condizentes com outros relatórios anteriores $(15,18,19,28)$, nos quais os autores descreveram que as raparigas apresentaram maior prevalência de excesso de peso (incluindo obesidade) na primeira infância.

Quanto à menor prevalência de excesso de peso e/ou obesidade que os indivíduos da ilha do Faial apresentaram em comparação com os da ilha de São Miguel, até onde sabemos, a presente publicação é a primeira a descrever este dado. Contudo, mais estudos são necessários para entender melhor as variáveis sociodemográficas que poderiam explicá-lo.

Sobre as faixas etárias, foram encontradas algumas relações estatísticas, principalmente em indivíduos de 4, 6 e 9 anos. Essas diferenças não seguem uma ordem cronológica e, por esse motivo, são difíceis de explicar. No entanto, também foi anteriormente encontrado em Portugal que, aos 4 e 7 anos, as raparigas apresentavam valores estatisticamente mais altos do que os rapazes e, aos 10 anos, os rapazes eram mais pesados que as raparigas (21). Esses resultados sugerem que é possível existir alguma relação entre excesso de peso e idade, principalmente aos 4, 6-7 e 9-10 anos.

Os resultados observados sugerem que as crianças são mais propensas a aumentar o seu IMC aos 4, 6 e 9 anos, o que nos pode indicar de que este é o melhor período para fortalecer as estratégias de prevenção da obesidade. É imperativo destacar a importância da prevenção e reforçar a necessidade de estratégias eficazes dedicadas à população em idade precoce de forma a controlar a prevalência de obesidade infantil.

\section{Conclusão}

As raparigas apresentaram valores de IMC mais altos que os rapazes aos 6, 7 e 9 anos. Também, as meninas apresentaram níveis mais altos de excesso de peso 
ences. Being female and having 4, 6 (only in boys) and 9 (both for girls and boys, separately) years of age were related with a higher risk of presenting overweight and/ or obese. Our results reveal a high prevalence of obesity mainly among female participants, heightening the need for interventions at early ages to effectively control childhood obesity in Portugal.

\section{Acknowledgements}

The authors acknowledge all the collaborators and participants involved in the study.

\section{Conflict of Interests}

All of the authors declare that no financial or personal relationship can be understood as representing a potential conflict of interest. e obesidade em comparação com os meninos, sem diferenças de idade. Ser criança do sexo feminino e ter 4, 6 (apenas nos rapazes) e 9 anos de idade (tanto para raparigas quanto para rapazes, separadamente) está relacionado com um maior risco de apresentar excesso de peso e/ou obesidade. Os nossos resultados revelam uma alta prevalência de obesidade principalmente entre participantes do sexo feminino, ressaltando a necessidade de intervenções nutricionais em idades precoces, de forma a controlar efetivamente a obesidade infantil em Portugal.

\section{Agradecimentos}

Os autores agradecem a todos os colaboradores e participantes envolvidos no estudo.

\section{Conflito de Interesses}

Cada um dos autores declara que não existe qualquer relacionamento financeiro ou pessoal que possa ser entendido como representando um potencial conflito de interesse. 


\section{References/ Referências}

1. Franks PW, Hanson RL, Knowler WC, Sievers ML, Bennett PH, Looker HC. Childhood obesity, other cardiovascular risk factors, and premature death. N Engl J Med. 2010 Feb 11;362(6):485-93.

2. Friedemann C, Heneghan C, Mahtani K, Thompson M, Perera R, Ward AM. Cardiovascular disease risk in healthy children and its association with body mass index: Systematic review and meta-analysis. BMJ. 2012 Sep 25;345(7876):e4759-e4759.

3. Spinelli A, Buoncristiano M, Kovacs VA, Yngve A, Spiroski I, Obreja G, et al. Prevalence of severe obesity among primary school children in 21 European countries. Obes Facts. 2019;12(2):244-58.

4. Park MH, Falconer C, Viner RM, Kinra S. The impact of childhood obesity on morbidity and mortality in adulthood: A systematic review. Obes Rev. 2012 Nov;13(11):985-1000.

5. Putnam C. Childhood overweight and obesity: <http://www.cdc.gov/obesity/childhood $>$. J Consum Health Internet. 2012;16(1):77-84.

6. Rankin J, Matthews L, Cobley S, Han A, Sanders R, Wiltshire HD, et al. Psychological consequences of childhood obesity: psychiatric comorbidity and prevention. Adolesc Health Med Ther. 2016 Nov; Volume 7:125-46.

7. Pulgarón ER. Childhood Obesity: A Review of Increased Risk for Physical and Psychological Comorbidities. Vol. 35, Clinical Therapeutics. 2013. p. A18-32.

8. Kershaw EE, Flier JS. Adipose tissue as an endocrine organ. In: Journal of Clinical Endocrinology and Metabolism. 2004. p. $2548-56$.

9. Ronti T, Lupattelli G, Mannarino E. The endocrine function of adipose tissue: An update. Vol. 64, Clinical Endocrinology. 2006. p. $355-65$.

10. Gulcelik NE, Usman A, Gürlek A. Role of adipocytokines in predicting the development of diabetes and its late complications. Endocrine. 2009 Dec 25;36(3):397-403.

11. Sabbatini AR, Fontana V, Laurent S, Moreno H. An update on the role of adipokines in arterial stiffness and hypertension. Vol. 33, Journal of Hypertension. 2015. p. 435-44.

12. Barseghian A, Gawande D, Bajaj M. Adiponectin and vulnerable atherosclerotic plaques. Vol. 57, Journal of the American College of Cardiology. 2011. p. $761-70$.

13. García-Hermoso A, Ceballos-Ceballos RJM, Poblete-Aro CE, Hackney AC, Mota J, Ramírez-Vélez R. Exercise, adipokines and pediatric obesity: A meta-analysis of randomized controlled trials. Vol. 41, International Journal of Obesity. 2017. p. 475-82.

14. De Onis M, Blössner M, Borghi E. Prevalence and trends of stunting among pre-school children, 1990-2020. Public Health Nutr. 2012 Jan 14;15(1):1428 .

15. Ng M, Fleming T, Robinson M, Thomson B, Graetz N, Margono C, et al. Global, regional, and national prevalence of overweight and obesity in children and adults during 1980-2013: A systematic analysis for the Global Burden of Disease Study 2013. Lancet. 2014 Aug 30;384(9945):766-81.

16. World Health Organization. Facts and figures on childhood obesity. Comm End Child Obes. 2014;14-6.

17. Rito A, Graça P. Childhood Obesity Surveillance Initiative: COSI Portugal 2013. 2015.

18. Rito AI, Paixão E, Carvalho MA, Ramos C. Childhood Obesity Surveillance Initiative: COSI Portugal 2010. 2012.

19. Wijnhoven TM, Van Raaij JM, Spinelli A, Starc G, Hassapidou M, Spiroski I, et al. WHO European Childhood Obesity Surveillance Initiative: Body mass index and level of overweight among 6-9-year-old children from school year 2007/2008 to school year 2009/2010. BMC Public Health. 2014 Aug 7;14(1):806.

20. Rito AI, Buoncristiano M, Spinelli A, Salanave B, Kunešová M, Hejgaard T, et al. Association between characteristics at birth, breastfeeding and obesity in 22 countries: The WHO European childhood obesity surveillance initiative - COSI 2015/2017. Obes Facts. 2019;12(2):226-43.

21. Freitas AI, Moreira C, Santos AC. Time trends in prevalence and incidence rates of childhood overweight and obesity in Portugal: Generation XXI birth cohort. Int J Obes. 2019 Feb 19;43(2):424-7.

22. Cole TJ, Bellizzi MC, Flegal KM, Dietz WH. Establishing a standard definition for child overweight and obesity worldwide: international survey. BMJ. 2000;320.

23. Garza JR, Pérez EA, Prelip M, McCarthy WJ, Feldman JM, Canino G, et al. Occurrence and correlates of overweight and obesity among island Puerto Rican youth. Ethn Dis. 2011;21(2):163-9.

24. Athanasopoulos DT, Garopoulou AI, Dragoumanos VP. Childhood obesity and associated factors in a rural Greek island. Rural Remote Health. 2011;11(4):1641.

25. Schwiebbe L, van Rest J, Verhagen E, Visser RWM, Holthe JK, Hirasing RA. Childhood obesity in the Caribbean. West Indian Med J. 2011 Jul;60(4):4425 .

26. Grech V, Farrugia Sant'Angelo V. Body mass index estimation in a school-entry aged cohort in Malta. Int J Pediatr Obes. 2009 Jan;4(2):126-8.

27. Curtis M. The Obesity Epidemic in the Pacific Islands. J Dev Soc Transform. 2004;1:37-42.

28. Pereira SA, Seabra AT, Silva RG, Katzmarzyk PT, Beunen GP, Maia JA. Prevalence of overweight, obesity and physical activity levels in children from Azores Islands. Ann Hum Biol. 2010 Oct 7;37(5):682-91.

29. World Medical Association Declaration of Helsinki. Ethical Principles for Medical Research. 2013; 310(20):2191-2192. Doi:10.1001/Jama.2013.281053

30. INSA. Childhood Obesity Surveillance Initiative COSI Portugal 2019. http://www.insa.min-saude.pt/cosi-portugal-2019-excesso-de-peso-e-obesidadeinfantil-continuam-em-tendencia-decrescente/ 